\title{
On the impossibility of temperature extraction from heavy ion induced particle spectra
}

\author{
Jens Konopka, Horst Stöcker, and Walter Greiner \\ Institut für Theoretische Physik, Postfach 1119 32, Johann Wolfgang Goethe-Universität, \\ D-60054 Frankfurt am Main, Germany
}

(August 22, 2018)

\begin{abstract}
Spectra of various particle species have been calculated with the Quantum Molecular Dynamics (QMD) model for very central collisions of $\mathrm{Au}+\mathrm{Au}$. They are compatible with the idea of a fully stopped thermal source which exhibits a transversal expansion besides the thermal distribution of an ideal gas. However, the microscopic analyses of the local flow velocities and temperatures indicate much lower temperatures at densities associated with the freeze-out. The results express the overall impossibility of a model-independent determination of nuclear temperatures from heavy ion spectral data, also at other energies (e.g. CERN) or for other species (i.e. pions, kaons, hyperons)!
\end{abstract}

Typeset using REVTEX 
The study of nuclear matter under extreme conditions is the basic motivation for heavy ion experiments [1.:2]. The aim is the creation of a large bulk of heated and compressed nuclear matter [3]. However, the temperature and the density are not straightforwardly determinated from these experiments. It is even not clear from the beginning, whether it makes sense to speak about a temperature, since the degree of equilibration in a single event is not a measurable quantity either. In this letter the relation between spectral information, which is measurable in experiments, and the density and the degree of random motion during the intermediate reaction stages is investigated using transport theory. The latter aspect is closely related to the question of thermalization and the possible analogy to thermostatic properties of heated and/or compressed matter.

Single particle and $N$-body transport models have been used extensively to gain insight into the stiffness of the equation of state, the strength of the momentum dependence and inmedium nucleon-nucleon scattering cross-sections [4 [8]. These calculations predict various observables in terms of different underlying assumptions. The question is, which of these aspects is dominating the dynamics of heavy ion collisions. This procedure is in fact very indirect. It would be more convenient to have a direct measure of thermodynamical quantities, like pressure or temperature, which could be connected to the properties of excited nuclear matter.

As an idealized test case, we performed QMD calculations [4, 5, 9] of a heavy system for $\mathrm{b}=0$, namely $\mathrm{Au}(150 \mathrm{MeV} /$ nucleon $)+\mathrm{Au}$. Such reactions are supposed to yield the largest accessible participant region, where the nucleons involved undergo a rapid sequence of binary collisions. Consequently, equilibrium, with a large number of nucleons involved, is more likely established in central collisions than in peripheral reactions or collisions of lighter partners.

In the present analysis no information other than mass, charge and four-momentum of the emitted products will be used for extraction of further information on the properties of the hot and dense nuclear matter formed in the transient state. Thus such an analysis would be applicable to experimental data as well [10,11]. 
After a full QMD propagation for $300 \mathrm{fm} / \mathrm{c}$, involving all nuclear interactions and hard binary scatterings, fragments are calculated via a $3 \mathrm{fm}$ configuration space coalescence. Then, in addition, all charged products are propagated on their mutual Coulomb-trajectories for another $10^{6} \mathrm{fm} / \mathrm{c} \approx 3 \cdot 10^{-18} \mathrm{~s}$.

Having obtained the full triple-differential spectra in such a manner for each charge independently, the spectra were fitted by a transversally expanding, thermally equilibrated source [12].

This implies that the transverse spectrum is composed of a directed flow and a random component, which can be associated with a temperature. The strength of the collective component is assumed to increase linearly with the transverse distance; the shape of the source is a homogeneous sphere. Nonrelativistically the double-differential distribution reads

$$
\begin{aligned}
\frac{\mathrm{d}^{2} f\left(T, p_{\max }\right)}{\mathrm{d} p_{\mathrm{l}} \mathrm{d} p_{\mathrm{t}}} & =N \frac{2}{p_{\max }} \int_{0}^{p_{\max }} \mathrm{d} p_{0} \frac{p_{0}}{p_{\max }}\left(\frac{\eta}{\pi}\right)^{\frac{3}{2}} 2 \pi p_{\mathrm{t}} \\
& \times \exp \left\{-\eta\left(p_{\mathrm{t}}^{2}+p_{\mathrm{l}}^{2}+p_{0}^{2}\right)\right\} I_{0}\left(2 \eta p_{\mathrm{t}} p_{0}\right)
\end{aligned}
$$

Here $\eta$ is an abbreviation for $\frac{A}{2 m_{N} T}$. With these formulas the average flow momentum per nucleon is $\frac{2}{3} p_{\max }$ and the averaged flow kinetic energy amounts to $p_{\max } / 4 m_{N}$.

In order to get information on the thermal collective energy sharing, we restrict our analysis for the moment to the transverse momentum spectra only. This procedure is motivated by the fact that transverse momenta are newly created and are not directly connected to the initial (longitudinal) beam momentum. A possible collective expansion in longitudinal direction cannot unambiguously be associated to the properties of the hot and dense reaction zone because the system may have memory about its history, in particular the incident momentum.

Fig. 11 shows transverse momentum spectra of various fragment charges obtained with QMD for the system $\mathrm{Au}(150 \mathrm{MeV} /$ nucleon, $\mathrm{b}=0)+\mathrm{Au}$ (symbols) together with fits to these calculated data, which are based on the assumptions from above. In fact, the corresponding count rates have been fitted, rather than the invariant distributions, which are displayed. All spectra are compatible with temperatures between 20 and $25 \mathrm{MeV}$ and averaged collective 
flow velocities of $0.1-0.13$ c. Fig. 2 shows the minimum of the $\chi^{2}$ distributions associated with the various fits, which exhibit a valley over a wide range of temperatures. For heavy fragments such as $\alpha$ 's or even heavier species, the minimum is well defined. It is represented in the by the bullet: For this class of probes the collective component can be extracted for this model in an unambiguous way: In the valleys of the $\chi^{2}$ distributions the corresponding flow momentum depends only weakly on the temperatures. On the contrary, for light particles like free protons and deuterons one observes that

1) the minimum is not as pronounced as in the case of the heavy composite fragments, particularly the fit for p's is of considerable minor quality and is not unique 2) the valley extends for p's like a banana from low temperatures with large flow to high temperatures with almost no flow with only a slight gradient. This implies that the heavy fragments clearly exhibit collective flow in these ultracentral collisions, while the flow cannot unambiguously be determined with light fragments. Collective flow is here associated with azimuthally symmetric collective expansion.

Fig. 2 shows the line corresponding to energy conservation When the fit procedure works well, the minimum of the $\chi^{2}$ distribution is in line with energy conservation. However, this is not the case for protons, which is a first indication that the proton emission pattern cannot be understood in terms of the simple ansatz of collective flow + thermal motion, although the proton spectrum in Fig. 11 seems to be properly fitted.

Fig. 2 indicates that the heavier fragments exhibit smaller averaged transverse collective expansion velocities: In the model these fragments form via the configuration space coalescence mechanism in the interior of the expanding nuclear matter. On the surface of the exploding system, where the local expansion velocity is highest, it is less probable to form heavy fragments, since the number density of nucleons around is much smaller than in the interior.

Let us summarize the analysis of the final spectra, apparently the momentum space population of all fragment species is reasonably well described by two parameters, the temperature $T$ and an averaged collective velocity $v_{c}$. The spectral information suggests tem- 
peratures larger than $20 \mathrm{MeV}$. Since this depends only on the final state of the collision, when all interactions on the nuclear length scale have ceased, it is concluded that the ashes of the exploding nuclear matter seem to indicate these high temperatures. The associated average collective velocity amounts to $10-15 \%$ of the speed of light. Quantitatively similar conclusions have been obtained from measured transverse momentum and global kinetic energy spectra in central collisions of $\mathrm{Au}+\mathrm{Au}$ at $150 \mathrm{MeV} /$ nucleon 10,13,14. However, a detailed analyses of the nuclear chemistry, i.e. the fragment abundances, in the very same experiment yields low temperatures of the order of $8 \mathrm{MeV}$ to account for the large fragment abundances 15. This has lead to speculations on different time scales for the chemical and the dynamical equilibration processes. The temperatures determined with the two methods, chemical determination from isotope ratios or fragment abundances on the one hand side and determination from the fragment spectral slopes on the other hand can not be reconciled in this scenario. We demonstrate below that this is due to misconceptions of the interpretation of yields and spectra in an instantaneous break-up of a homogeneous, globally equilibrated system.

QMD allows for a microscopic calculation of all quantities relevant to the reaction dynamics and the nuclear thermodynamics. The time evolution of the composite system is followed in more detail [12] by dividing configuration space in various cells on a cylindrical grid. For each of these cells the mean and the spread of the local velocity distribution is calculated for a superposition of many events, obtained under the same macroscopic conditions. The mean velocity illustrates the collective velocity of each individual cell, whereas the spread around the mean reveals the thermal, i.e. random, motion inside the cell. The directional dependences of the temperature parameters (longitudinal, transversal, and azimuthal temperatures $T_{\|}, T_{\perp}, T_{\varphi}$ ) have also been extracted. They correspond to the widths of the local velocity distribution in the directions parallel to the beam, perpendicular to the beam, and in azimuthal direction. These values are equal in each cell in the case of local equilibrium. If the temperatures were, in addition, constant over the full volume, this would be global thermal equilibrium. The complete information on the local collective velocities, 
and on the degree of randomness is available. It tests our initial assumption of a linearly increasing flow velocity profile and we can analyze to which extend global or local thermal equilibrium is established in the model.

In the following we characterize the central reaction zone in terms of the global parameters density and temperature, which are important for the (multi-)fragmentation of the source. The central reaction zone is defined here as the volume of all cells with density at least half of the maximum density at this instant. This definition implies a time-dependent volume, which is considered here.

A considerable fraction of the nucleons remains in the central volume (Fig. 3 a)): even after $100 \mathrm{fm} / \mathrm{c}$ about one third of the system is still in the central zone. Matter outside this zone has experienced the higher densities earlier on. In terms of a hydrodynamical point of view, matter in the QMD model freezes out at different times. The temperature distributions indicate rapid cooling and prove that the source of nucleons and clusters is not globally equilibrated, even for the most central collisions in microscopic models (Fig. 3 b)). However, in the longitudinal direction the spread of the velocity distribution is higher by $50 \%$ in the intermediate reaction stages $(t=40-60 \mathrm{fm} / \mathrm{c})$, while the system is transversally equilibrated. This cooling is associated with a decrease of the density too (Fig. 3 c)).

Fig. 4 shows that at low densities $\left(<0.5 \rho_{0}\right)$, where the fragment freeze-out is commonly assumed to occur, the microscopically determined true (local) temperatures (symbols) have dropped below $10 \mathrm{MeV}$. Thus they strongly deviate from the slope temperatures extracted from the simple macroscopic fit to the (microscopically calculated) fragment spectra (light grey shaded area). The values compare reasonably well with curves of constant entropy obtained with the quantum statistical model (QSM) [16] and with a QSM analysis of experimental fragment yields [15] (dark shaded area).

This explanation of the large discrepancy between the temperatures extracted from the chemical analysis and the temperatures extracted from the fragment spectra puts into question all temperature extractions from slopes of particle spectra, even if an additional collective motion is taken into account. This was done, for example, in our macroscopic analysis 
above. The values are 2-3 times higher than the correctly extracted microscopic values. This finding concerns also temperatures extracted from slopes of pion spectra at highest incident energies, e.g. at the AGS and CERN [17].

What is wrong with the assumptions underlying the global fit? A thermally equilibrated source at some temperature $T$ can disintegrate not only due to the thermal pressure, but also due to some additional collective expansion. The probability of finding a particle which has received a collective velocity $v_{\text {coll. }}$, i.e. $d N / d v_{\text {coll. }}$, is essentially unknown. It can be expressed as

$$
\frac{\mathrm{d} N}{\mathrm{~d} v_{\text {coll. }}}=\frac{\mathrm{d} N}{\mathrm{~d} r} \cdot \frac{\mathrm{d} r}{\mathrm{~d} v_{\text {coll. }}}=\frac{\mathrm{d} N}{\mathrm{~d} r} \cdot\left(\frac{\mathrm{d} v_{\text {coll. }}}{\mathrm{d} r}\right)^{-1}
$$

Hence, the density distribution as well as the flow velocity profile enters as the first and second term on the right hand side of the preceding equation. For a fit to the spectra, a homogeneous density with a sharp cut-off and a linearly increasing velocity profile have been used [18].

Therefore, the high transverse momenta components of the particle spectra do not correspond to the high momentum tails of hot source, but to the collective motion of fast cells with low internal temperature. The microscopic analysis suggests that the combinations of density and temperatures, which are traversed in the course of the reaction, are in agreement with the expectations from the quantum statistical analysis of the fragment distributions in the final state 15 .

In summary, temperatures obtained from slopes of particle spectra can only serve as an upper estimate for the conditions at freeze-out, even if collective flow has been taken into account. The main origin of this uncertainty is the fact that the configuration space distribution is essentially unknown. In view of this analysis, model estimates of temperatures in high-energy and ultrarelativistic heavy ion collisions [17] are expected to overestimate the thermal energy reached in such reactions considerably. Our analysis suggests that chemical thermometers, e.g. $\pi / p$ ratios, which take into account feeding from unstable states and absorption may be more appropriate. 


\section{ACKNOWLEDGEMENT}

This work was supported by BMFT, DFG, and GSI. 


\section{REFERENCES}

[1] H. Stöcker and W. Greiner, Phys. Rep. 137, 277 (1986).

[2] R. B. Clare, D. Strottmann, Phys. Rep. 141, 177 (1986).

[3] Proceedings on the NATO Advanced Study Institute Programme on Hot and Dense Nuclear Matter, eds. W. Greiner, H. Stöcker, and A. Gallmann, NATO ASI Series B Vol. 335, Plenum, N.Y. (1994).

[4] J. Aichelin et al., Phys. Rev. C 37, 2451 (1988).

[5] G. Peilert et al., Phys. Rev. C 39, 1402 (1989).

[6] C. Gale et al., Phys. Rev. C 35, 1666 (1987).

[7] B. Blättel et al., Rep. Prog. Phys. 56, 1 (1993).

[8] A. Faessler, Prog. Part. Nucl. Phys. 30, 229 (1993).

[9] G. Peilert et al., Phys. Rev. C 46, 1457 (1992).

[10] J.P. Coffin et al, Proc. of Nucleus-Nucleus Collisions V, Taormina, Italy, 1994, eds. M. di Toro, E. Migneco, and P. Piattelli, Nucl. Phys. A 583 567c (1995).

[11] M. A. Lisa et al., LBL-Preprint 35504 UC-414 (1994).

[12] J. Konopka et al., Proc. of Nucleus-Nucleus Collisions V, Taormina, Italy, 1994, eds. M. di Toro, E. Migneco, and P. Piattelli, Nucl. Phys. A 583 357c (1995).

[13] S.C. Jeong et al., Phys. Rev. Lett. 72, 3468 (1994).

[14] W. Reisdorf for the FOPI-Collaboration, Proceedings of the XXII Workshop on Gross Properties of Nuclei and Nuclear Excitations, Hirschegg 1994, p. 93.

[15] C. Kuhn et al., Phys. Rev. C 48, 1232 (1993).

[16] D. Hahn and H. Stöcker, Nucl. Phys. A476 718 (1988). 
[17] E. Schnedermann and U. Heinz, Phys. Rev. Lett. 69, 2908 (1992).

[18] H. Stöcker et al., Z. Phys. A 303, 259 (1981). 


\section{FIGURES}

FIG. 1. Invariant transverse momentum distributions of various charged fragments emerging from $\mathrm{Au}(150 \mathrm{MeV} /$ nucleon, $\mathrm{b}=0)+\mathrm{Au}$ collisions. The lines indicate fits to the calculated points involving a azimuthally symmetric, transversally expanding, and thermally equilibrated source. The fits have been performed for each fragment specie independently. The associated count rates rather than the displayed invariant distributions have been fitted. All spectra are compatible with a temperature between 20 and $25 \mathrm{MeV}$ and averaged collective flow velocities between 0.10 and $0.13 \mathrm{c}$.

FIG. 2. $\chi^{2}$ distributions for the two parameter fit to the spectra in Fig. 1. Contourlines are at $2,3,4$, and 5 times the minimum value. The minima are indicated as black circles. The thick solid lines correspond to those pairs of parameters where the total kinetic energy is conserved. For

the case of protons, the quality of the fit in terms of $\chi^{2}$ is poor. The energy constraint tends to predict lower temperature and larger flow.

FIG. 3. Thermodynamics in the central reaction zone, i.e. the volume where the local density is at least half of the maximum value. Mass content a), spreads of the local velocity distributions b), and maximum as well as averaged density c) are displayed at a function of time.

FIG. 4. Temperatures versus densities for central $\mathrm{Au}+\mathrm{Au}$ collisions at $150 \mathrm{MeV} /$ nucleon. The microscopically determined local temperatures (symbols as in Fig. 3) are considerably lower than the simple macroscopic fit to the spectra (light grey shaded area). However, they compare reasonably well with curves of constant entropy from QSM and with experimental data from a quantum statistical analysis of the fragment distributions (dark grey shaded area) [15]. 


\section{$\mathrm{Au}(150 \mathrm{MeV} /$ nucleon, $\mathrm{b}=0)+\mathrm{Au}$}

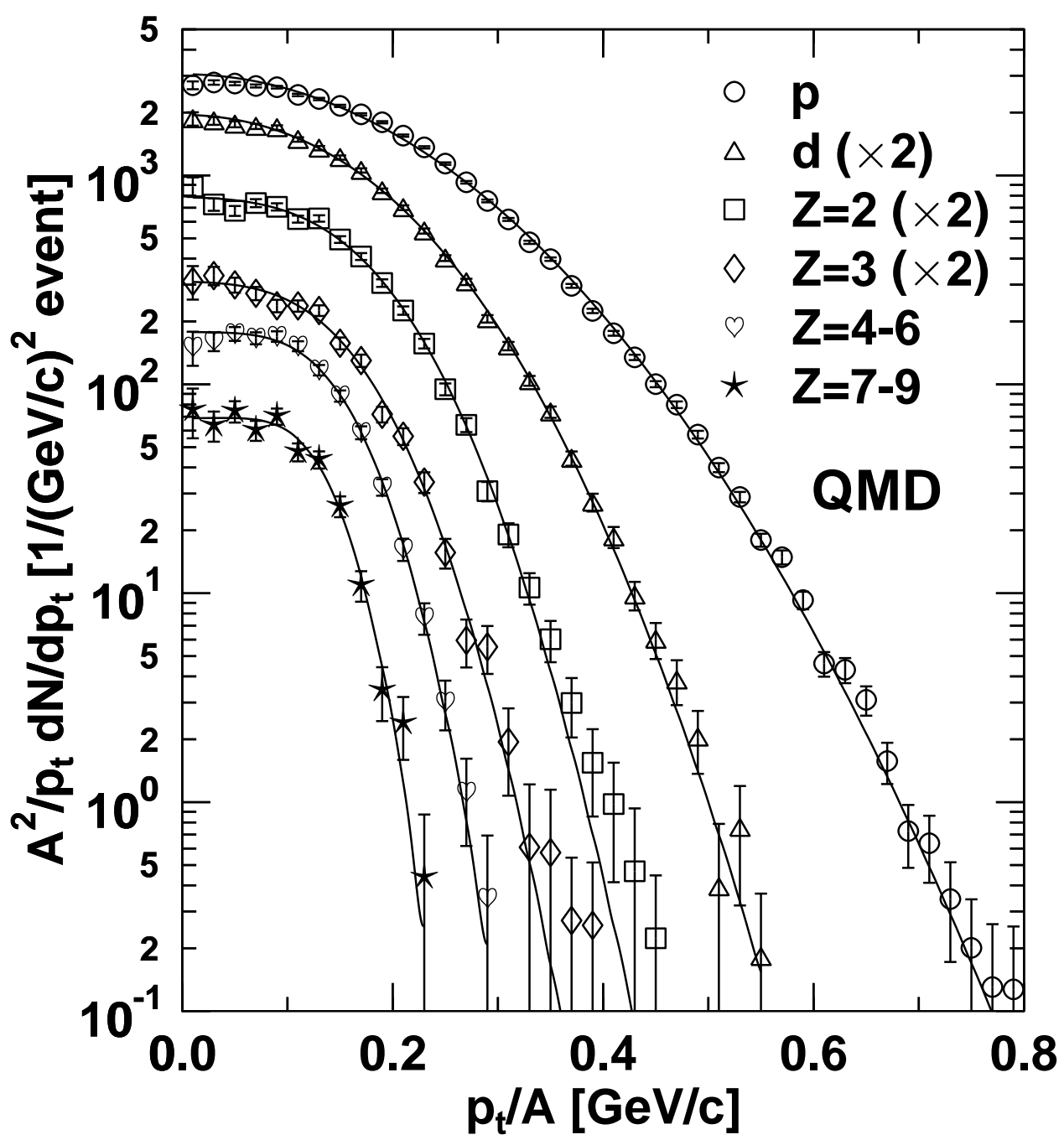

J. Konopka et al., Fig. [1 


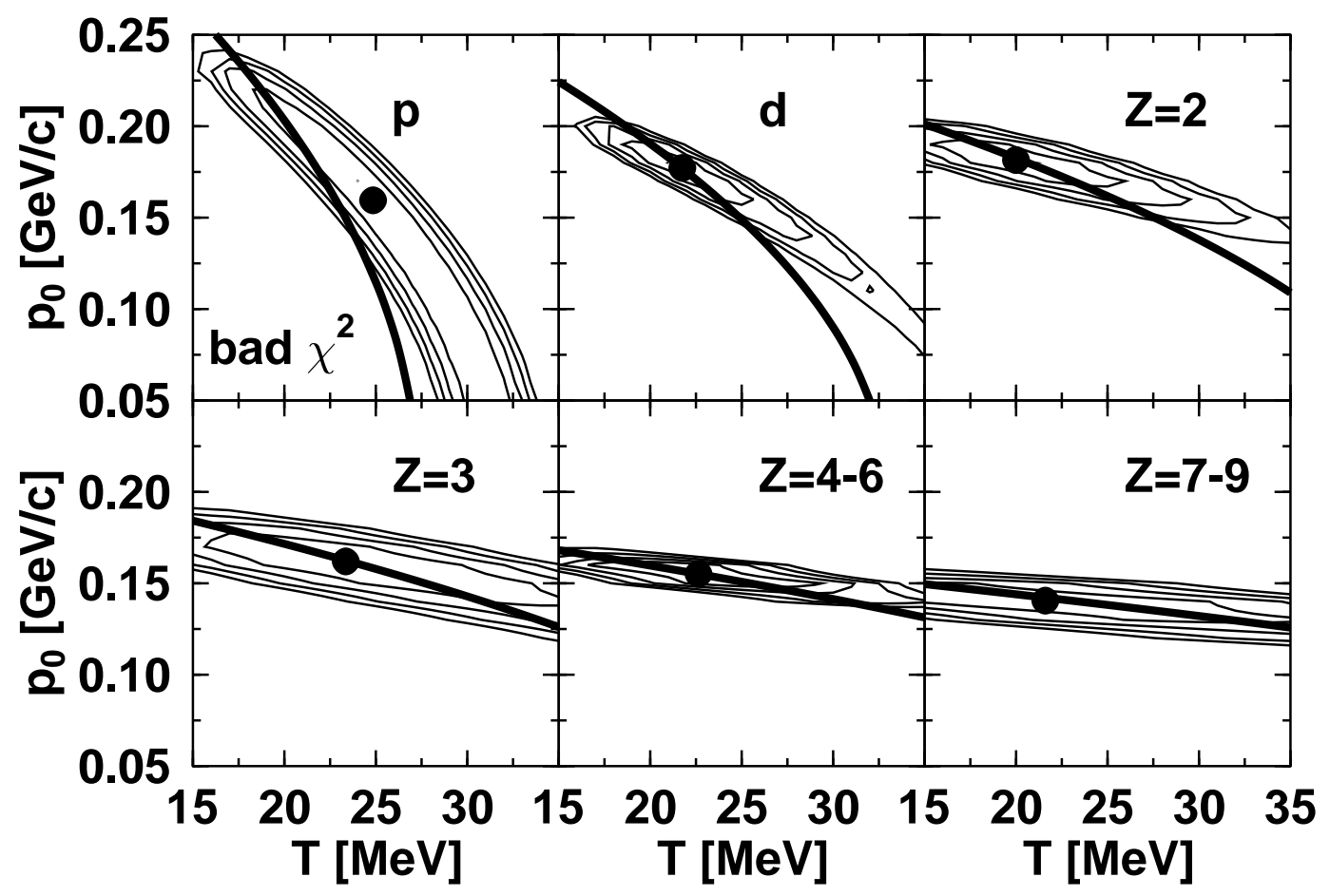

J. Konopka et al., Fig. 2 


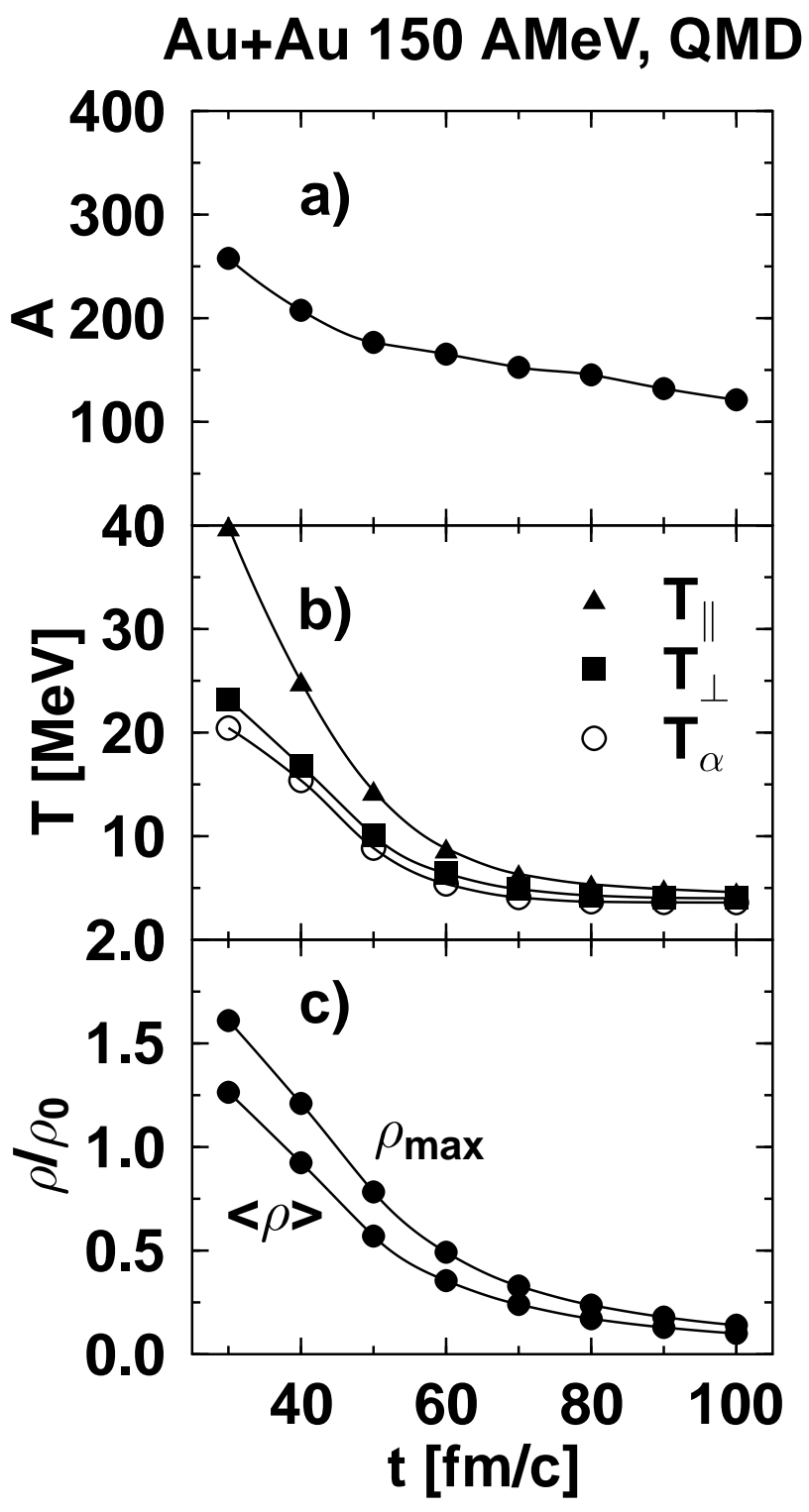

J. Konopka et al., Fig. 3 


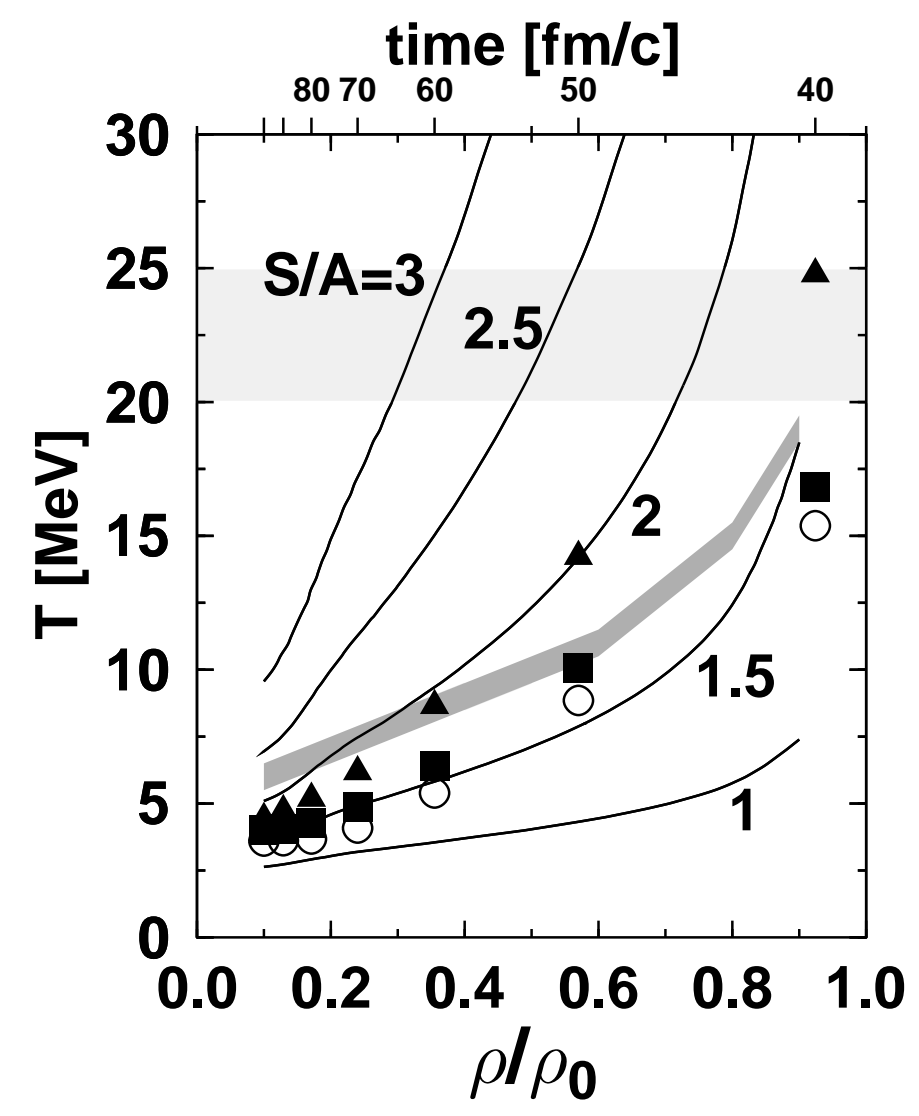

J. Konopka et al., Fig. 田 